\title{
Rapid 3D Reconstruction in the EDS Tomography by using Iterative Series Reduction (ISER) Method.
}

\author{
Yoshitaka Aoyama ${ }^{1}$, Hideo Nishioka ${ }^{1}$ and Yukihito Kondo ${ }^{1}$ \\ 1. JEOL Ltd., 1-2 Musashino 3-Chome Akishima Tokyo 196-8558 Japan
}

Energy Dispersive X-ray Spectroscopy (EDS) is widely used to obtain the elemental maps of a sample. EDS Tomography is a method to reconstruct three-dimensional (3D) elemental maps from a set of twodimensional (2D) EDS elemental maps. The maps can be obtained by a transmission electron microscope (TEM) equipped with EDS detector. The high accelerating voltages of TEM is necessary to obtain the transparent image and elemental maps. On the other hand, many 2D elemental maps, forming a tilt series map, are necessary to reconstruct 3D elemental maps. Therefore, the electron beam damage is serious in EDS Tomography, and a method to reduce the number of elemental maps is sought-after.

It is well known that there are two types of algorism to reconstruct the 3D structure. One is the analytical method like Filtered Back Projection method (FBP), and another is the algebraic method like Simultaneous Iterative Reconstruction Technique (SIRT). Iterative Series Reduction (ISER) is a new algebraic method for 3D reconstruction based on the sparse modeling technique, which is rapidly developing in the field of the image processing [1] [2]. By using ISER, 3D reconstruction can be obtained from only several projections. In this method, only the outlines of the objects in the projections are used in the 3D reconstruction to make the sparse model. The other regions are ignored in the 3D reconstruction. This reconstruction is achievable with small number of the variables. Therefore, by using ISER, small number of the sparse modeled projections is enough to reconstruct the 3D structure, if one only wants to know the outline of the objects. It is useful to determine the shape of the sample, even if the gradient of the density in the specimen cannot be reconstructed.

In this study, we tried to combine EDS Tomography and ISER to shorten the acquisition time. This avoids the beam damage and contamination of the sample. Figures 1 show the BF-STEM image of a paint film and elemental maps of Titanium, Aluminum, Iron and Silicon. A tilt series of EDS elemental maps for the region of sample shown in Fig. 2 was collected with the tilting range of \pm 56 degree. The interval of the tilt steps was 8 degree. The number of pixels for these maps was $256 \times 256$. The number of the elemental maps was only 15. Figure 2 shows the 3D elemental maps of the paint film reconstructed by ISER. Figure 2(a-c) shows the orthogonally-cut digital slices from the 3D reconstructed map of Titanium. The spurious streaks in the recorded tomograms (not shown here) due to the missing wedge of rotation were disappeared in digital slices shown in Fig. 2(a-c). Figure 2(d) shows the volumerendering image of the paint film. The brown, green, pink and yellow phases correspond to the Titanium, Silicon, Aluminum and Iron. In conclusion, using ISER for EDS Tomography reconstruction, we can reduce number of elemental maps to be 15 , which is generally $\geqq 50$ by the conventional method per a tilt series for 3D reconstruction. This greatly reduces the beam irradiation damage of specimens and opens the possibility for $3 \mathrm{D}$ reconstruction of beam sensitive materials.

\section{References:}

[1] D L Donoho, IEEE Trans. Inf. Theory 52 (2006) p. 1289-1306.

[2] E J Candes, J Romberg and T Tao, IEEE Trans. Inf. Theory 52 (2006) p. 489-509. 


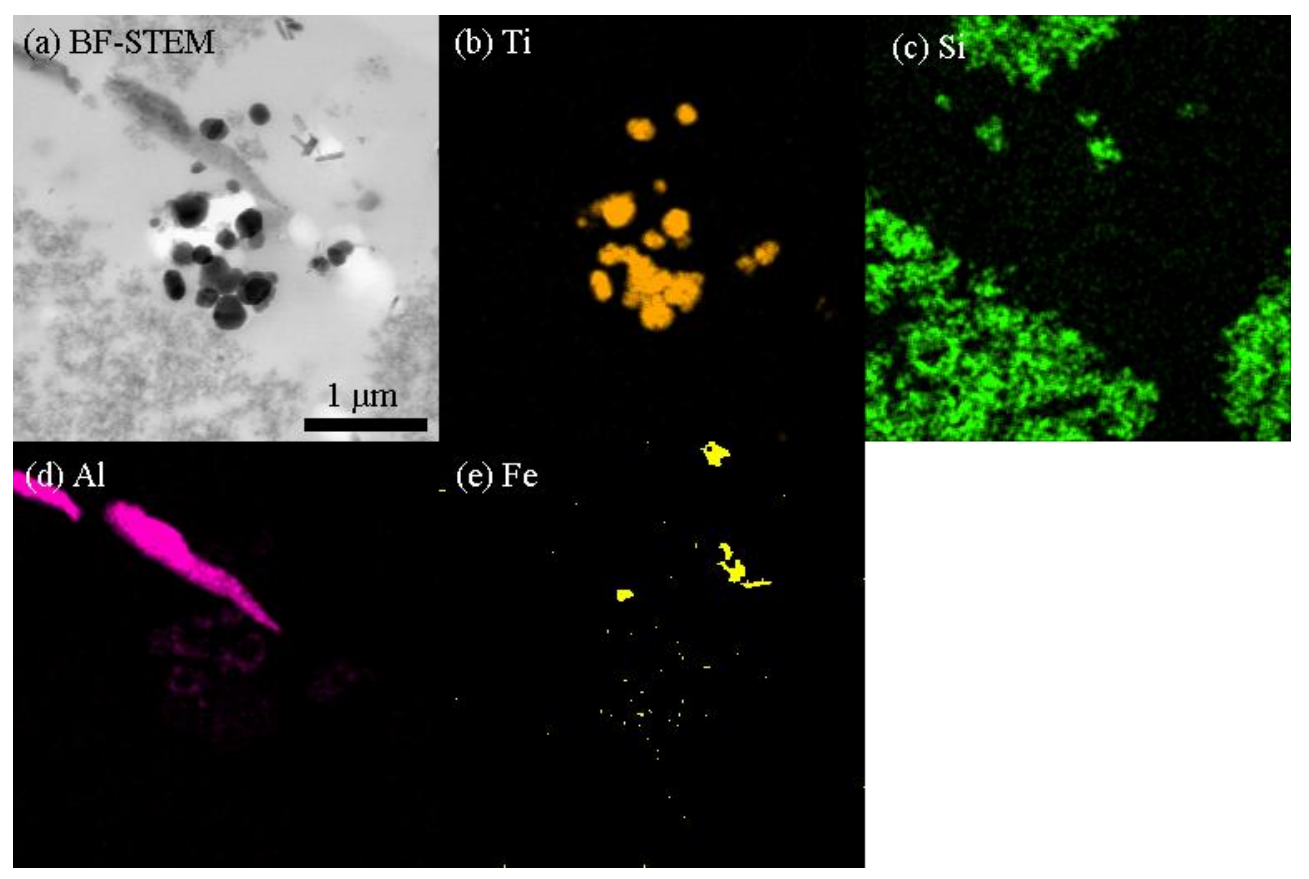

Figure 1. BF-STEM image and elemental maps of the paint film. (a) BF-STEM image, (b) Titanium map, (c) Silicon map, (d) Aluminum map and (e) Iron map.
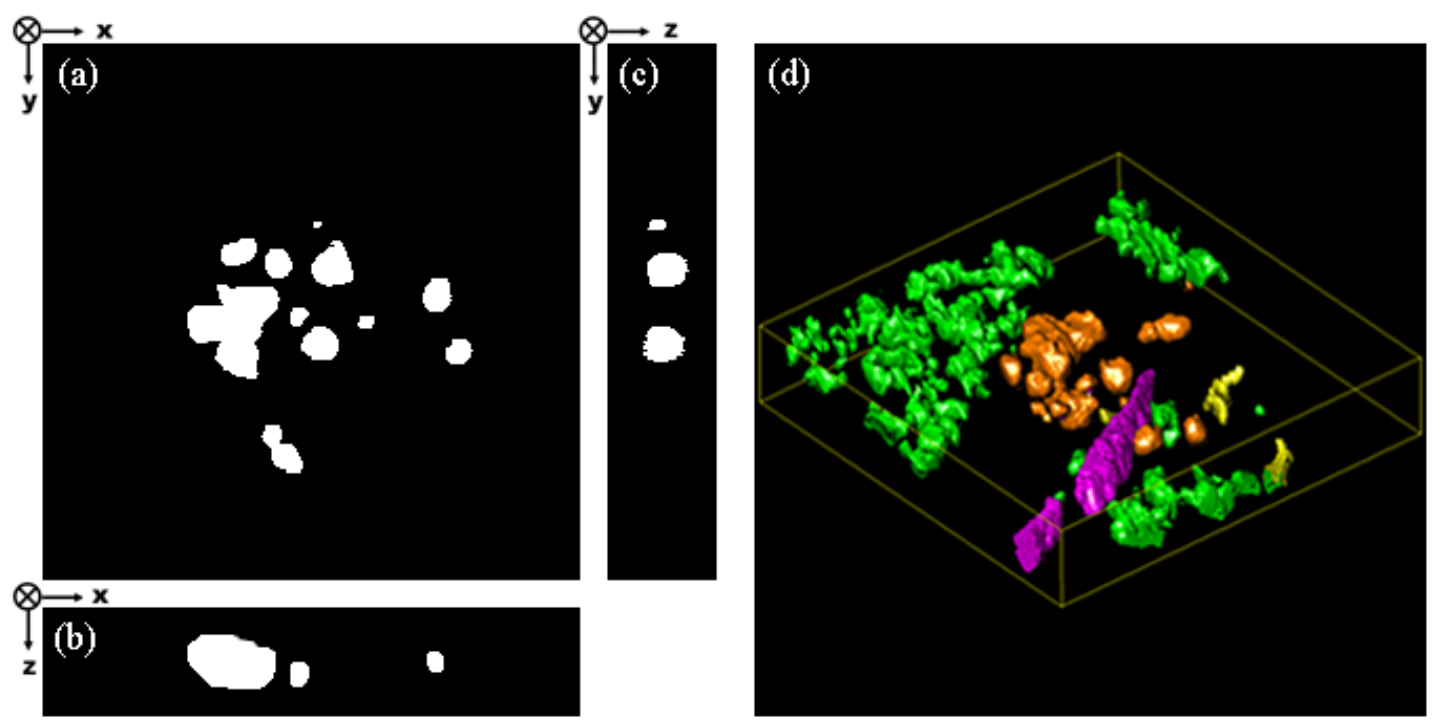

Figure 2. (a) The $3 \mathrm{D}$ reconstruction of the Titanium from the paint film was obtained by ISER. The interval of tilt steps was 8 degree. The tilting angular range was \pm 56 degree. (b) The volume-rendering image of the paint film. The brown, green, pink and yellow phases correspond to Titanium, Silicon, Aluminum and Iron. 